\title{
From promise to practice
}

\author{
The role of synthetic biology in green chemistry
}

\author{
David R. Nielsen \& Tae Seok Moon
}

$\mathrm{T}$ he work by the French chemist Antoine Lavoisier in the late eighteenth century on the law of conservation of matter and the oxygen theory of combustion helped to ignite a 'chemical revolution' that shifted the field of chemistry from observational studies to synthesis applications. The improved understanding of chemical reactions and the increasing ability of chemists to synthesize compounds ushered in a new era of synthetic chemistry that helped to drastically improve quality of life through synthetic fertilizers, pesticides, medicines, plastics and dyes and that spawned new industries on which many nations built their modern economies. Today, the emerging field of synthetic biology finds itself at a similar precipice: it has the potential to repeat the successes of chemistry by shifting biology from a research field largely based on observation to a discipline that emphasizes design and construction of novel biological systems and organisms. Coming full circle with history, one of the first fields that will benefit from synthetic biology is chemistry.

Although synthetic chemistry and the chemical industry have drastically improved human quality of life, they have also created massive problems in terms of environmental pollution, toxic waste and even endangering public health. The growing environmental awareness and the rising costs for the prevention or cleaning up of environmental damage spawned the concept of 'green chemistry', which is the design of products and synthetic processes that minimize or prevent the use and generation of toxic

\section{... one of the first fields that will benefit from synthetic biology is chemistry}

compounds and reduce requirements for energy and natural resources.

$N$ ature has helped to achieve this goal. Billions of years of evolution have generated an enormously diverse natural product portfolio and associated enzyme chemistries. Synthetic biologists are just beginning to exploit this wealth to design renewable chemicals and production processes that minimize pollution at the source, which is an underlying goal of green chemistry. Compared with traditional industrial chemical synthesis, biosynthetic processes offer several key advantages. Generally speaking, enzymes most efficiently and commonly work under biologically relevant conditions_ambient temperatures and pressures, aqueous media and neutral $\mathrm{pH}$-enabling a multitude of diverse enzymes to work simultaneously within the same space, such as an organelle or a cell. This makes it possible to assemble multistep synthetic pathways for the in vivo production of complex molecules from cheap and renewable substrates (Fig 1). Unlike most multistep processes in chemical synthesis, biosynthetic pathways do not require recovery, purification, and further processing of intermediate products. For example, as enzymes are highly specific for their substrates, it is not necessary to protect or de-protect other parts of the molecule to prevent unwanted side reactions.

These features collectively enable 'onepot synthesis', which is rare in chemistry. Biosynthesis would also obviate the need for harsh and energy-intensive reaction conditions, replace toxic solvents and caustic reagents with milder alternatives, and substitute toxic catalysts (often heavy metals) for benign and biodegradable biocatalysts. Bio-based chemicals would also reduce greenhouse-gas emissions and costs through the use of cheaper substrates including non-food plants, agricultural and municipal waste and even atmospheric $\mathrm{CO}_{2}$. 'Greener' chemicals and processes would therefore assuage the short-term and long-term environmental effects of the chemical industry. There is an economic benefit as well. By 2020, 'green chemistry' practices are projected to save the petrochemical and fuel industries up to US\$65.5 billion through direct cost savings and indirect savings associated with, for example, avoiding liability for environmental damage (http://www.navigantresearch. com/newsroom/green-chemical-industryto-soar-to985-billion-by2020).

\section{The growing environmental awareness and the rising costs for preventing or cleaning up environmental damage spawned the concept of 'green chemistry'...}

'Green chemistry' continues to grow thanks largely to the maturing practices by which micro-organisms can be engineered as biocatalysts to convert renewable feedstocks into petrochemical replacements. The focus of many researchers in industry and academia, however, has shifted from biofuels to biochemicals. The motivation is price: bulk and fine chemicals have higher values than fuels per unit mass and serve smaller markets. Although the chemicals market uses only $5 \%$ as much carbon as the fuel market, its net economic value is nearly equal [1]. Thus, creating 'greener' and renewable alternatives to conventional petrochemicals is economically more promising than development of biofuels. 


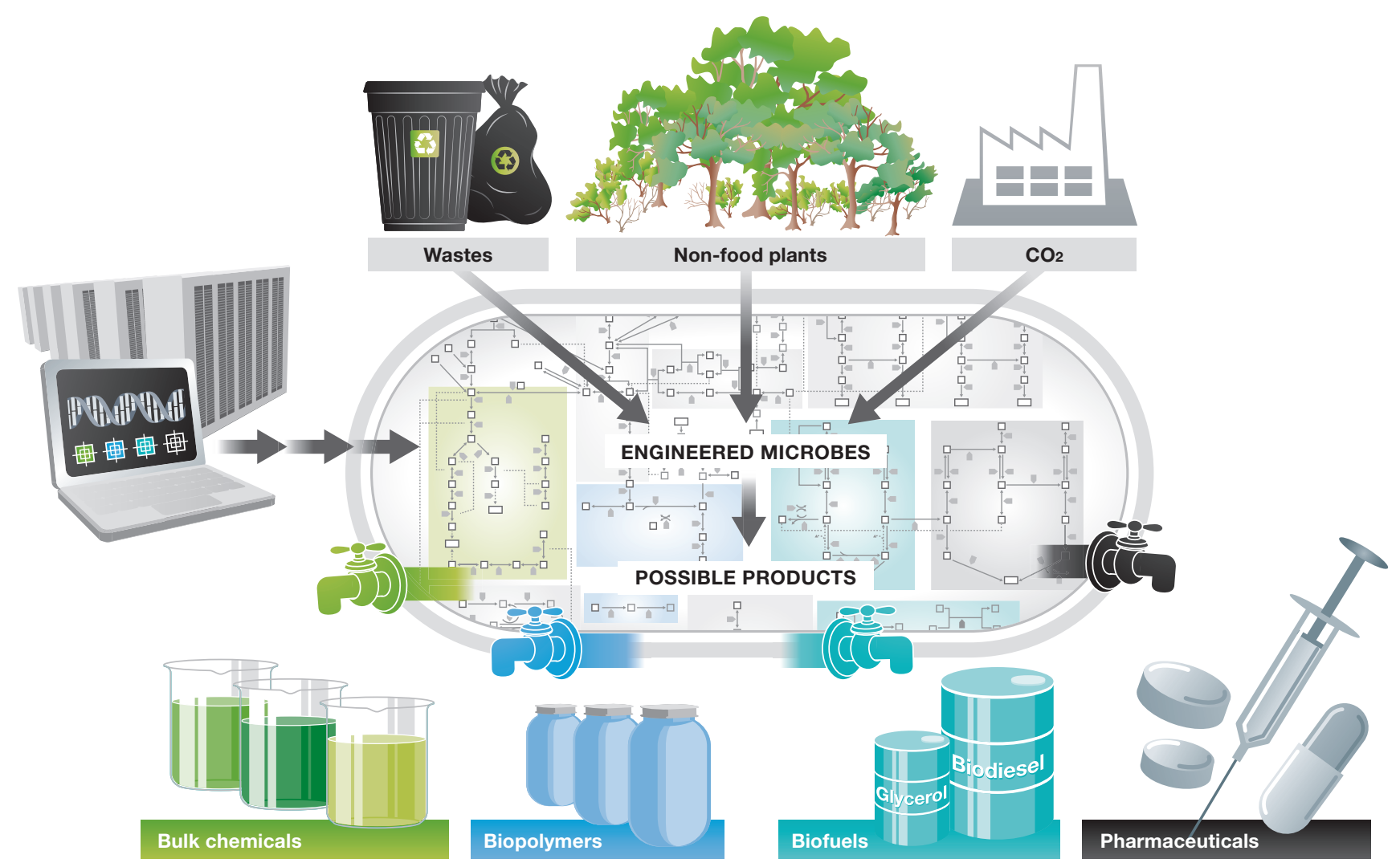

Fig 1 The potential of synthetic biology to contribute to green chemistry. Engineered organisms could synthesize a range of important chemicals from renewable resources, waste and, potentially, carbon dioxide.

W hen engineering micro-organisms to produce a target compound, synthetic biology and metabolic engineering - two related fields that intersect at the level of metabolicpathway construction - are key to enabling biocatalyst engineering and maximizing cellular productivity. Metabolic engineering has a long track record in 'green chemistry' and has traditionally focused on optimizing metabolic flux through natural pathways in native or heterologous host organisms. These improvements have enabled the production of numerous naturally occurring bioproducts at near theoretical yields, including, for example, the plastics monomers succinic acid and lactic acid, and $n$-butanol, a solvent and next-generation biofuel.

In contrast, a key contribution of synthetic biology is the engineering of living factories', which can produce chemicals that were previously unattainable, previously attainable only via expensive extractions or that have never been synthesized in nature. For example, engineered microbial biocatalysts with de novo created biosynthetic pathways now produce styrene and 1,3-propanediol, which are both bioplastic monomers. Just as organic chemists use retrosynthetic schemes to synthesize the desired target compound, synthetic biologists use retrobiosynthetic schemes to design and construct metabolic pathways [2]. They exploit the natural diversity of enzymes to create enzymatic cascades that direct renewable feedstocks towards specific products of interest.

The central tenets of synthetic biology are modularity and standardization, which allow engineers to create biological 'parts' and 'devices' that can be rationally recombined in systems. Current efforts seek to expand the catalogue of available parts, devices and systems and to improve the ability by which synthetic biologists can combine and integrate them. Roughly speaking, parts are genetic elements with basic biological functions. In retrobiosynthetic applications, for example, enzyme-encoding genes can be recruited and expressed as needed to construct the designed synthetic pathway. Other parts, including promoters and ribosome-binding sites, allow quantitative control of gene expression and optimum flux of metabolites through the engineered pathways.
These parts enable the engineer to construct devices to achieve specific, defined functions. These include, for example, gene cassettes that express engineered pathways, or that enable the use of alternative, non-natural sources of carbon and/or energy-such as rare or complex sugars, or even $\mathrm{CO}_{2}$-and operons that enhance tolerance to inhibitory chemical products. Devices have also been engineered to improve target-product titres by, for example, providing dynamic control over the expression of key pathway enzymes in response to metabolic changes $[3,4]$. Finally, systems combine multiple devices whose coordinated functions allow a host or 'chassis' to simultaneously execute multiple programmed tasks. Meanwhile, the development of several generalizable approaches to global optimization of chassis is helping to maximize the productivity of engineered systems.

$\mathrm{T}$ he paradigm of retrobiosynthetic pathway engineering has allowed synthetic biologists to engineer novel routes that push the boundaries of nature's known biosynthetic capabilities. However, 


\section{By 2020, 'green chemistry' practices are projected to save the petrochemical and fuel industries up to US $\$ 65.5$ billion...}

there are still limits in regard to the products that can be synthesized, owing to a lack of known enzymes that are capable of catalysing the desired chemistries. Enzyme engineering, bioinformatics and computational biology collectively address this bottleneck to find or create enzymes with novel functions and expanded capabilities.

First, enzymes can be altered to modify or expand their native substrate specificity. Directed evolution and DNA shuffling are often the first choices for synthetic biologists to engineer enzymes. Second, nature itself remains a largely untapped resource for biocatalytic diversity. Metagenomics already uses genomics to bypass the need to isolate and cultivate individual species from vast microbial communities. Inspired by metagenomics, synthetic biologists have proposed the use of 'synthetic metagenomics' for parts discovery [5]. This approach uses bioinformatics to search sequence databases for putative biocatalytic functions; DNA synthesis and screening would then lead to isolation of new enzymes. Last, in silico design of enzymatic activities has already been demonstrated: de novo computational design of enzymes could soon expand the library of achievable enzyme function. These complementary approaches will ultimately lead to a larger catalogue of enzymes, and limited enzyme availability will no longer hinder the imagination of pathway designers.

Retrobiosynthesis is a powerful strategy for targeting specific end products, but what about products that we do not yet know we want? Billions of years of evolution have resulted in a hugely diverse portfolio of natural product pathways that could include new cures to modern diseases or that could serve as superior biofuels. A vast number of these pathways remain undiscovered, because the traditional discovery process is intrinsically limited: more than $99 \%$ of bacterial strains are difficult or impossible to culture; biosynthetic genes in organisms that can be cultured are often silent under laboratory conditions; and the cues that trigger gene expression are usually unknown.

Recent advances in genome sequencing, computational biology and DNA synthesis are about to fundamentally change the discovery process. First, the rapidly growing volume of genomic and metagenomic sequence data is a rich resource for mining not only single enzyme parts but also entire gene clusters involved in natural product biosynthesis, for example in the case of polyketides and non-ribosomal peptides. Gene clustering allows the rapid identification of pathway genes without a priori knowledge of non-cultivable strains' abilities to produce natural products. Second, new computational tools assist in the genome-wide prediction of natural product gene clusters, which helps to automate the genome-mining process. Last, pathway refactoring provides a systematic approach to activate and regulate pathway gene clusters in heterologous hosts. DNA is recoded without changes to its function while native regulation is removed and synthetic controls are implemented. Combined with rapid DNA synthesis, this approach has enabled the high-throughput screening of natural product pathways bypassing the cell's regulatory pathways. Together, such tools and strategies provide new ways to discover previously uncharacterized natural biosynthetic functions and, in doing so, lead to the discovery of new and useful chemical products.

W hile the search for new enzyme genes and product pathways continues, implementation of these into cells as biological factories is not straightforward and raises various problems. The introduction of non-native, synthetic and even xenobiotic pathway elements and molecules often disrupts the host organism's central metabolism. Common perturbations include side reactions by promiscuous heterologous enzymes, toxic effects of intermediates and products, competition for metabolites and energy resources, and the metabolic burden associated with synthesizing sufficient amounts of the desired chemical. Thus, to achieve functionality of a newly engineered or discovered pathway is merely the first step in the production of novel 'green chemicals'. Next in the queue for synthetic biologists is the challenge of maximizing yields, titres, productivity and host tolerance.

...there are still limits in regard to the products that can be synthesized, owing to a lack of known enzymes that are capable of catalysing the desired chemistries
Many enzymes are not highly specific catalysts of just one reaction but are promiscuous: approximately $40 \%$ of the metabolic enzymes of Escherichia coli accept multiple substrates and are responsible for catalysing of $65 \%$ of its metabolic reactions [6]. Although enzyme promiscuity enables the engineering of parts with new catalytic functions, it can also lead to unwanted and detrimental side reactions. Thus, eliminating crosstalk between synthetic metabolic pathways and the native metabolic network remains a challenging task in synthetic biology.

\section{Additional and substantial investment of time and money are still required for synthetic biology to reach the stature of a 'mature discipline'}

To function effectively and collectively, synthetic parts and devices must be engineered for mutual compatibility without affecting the performance of others parts. Such orthogonality can be easily achieved in non-biological production system, for example by using separate reactors to perform a series of chemical reactions and to prevent cross-reactions between intermediates. In biological systems, however, orthogonality is difficult to implement given that a vast range of reactions and interactions occur simultaneously within a cell. The introduction of even just a single heterologous gene can therefore cause severe perturbations. Metabolite channelling offers a potential solution by confining synthetic pathway enzymes to localized 'assembly lines' made of proteins, RNAs or DNAs. Alternatively, physical barriers can be created to confine metabolic pathways to distinct intracellular compartments for example membrane-bound organelles or bacterial protein shells. Both strategies can be useful for eliminating unwanted interactions in engineered biocatalysts.

The relentless push towards economical titres, yields and productivity means that product toxicity often becomes a critical limiting factor, particularly for novel chemicals that do not occur in the natural environment. If microbes have not previously been exposed to such molecules throughout their evolutionary timeline, they typically lack the machinery required for tolerance. However, effective strategies 
for improving tolerance have been developed by focusing on what is perhaps the most common location for detrimental interactions between microbes and hydrocarbon products: the inner membrane, where the accumulation of 'solvent-like' products reduces membrane integrity and function. The structure can be fortified by alteration of its lipid composition and content. Alternatively, inhibitory products can be exported from the cell via active efflux pumps and other transporters.

$\mathrm{T}$ he discovery and development of new biosynthetic pathways and the engineering of robust microbial production systems are already expanding the number and diversity of chemical products that can be synthesized from renewable resources, including drugs, building-block molecules, monomers and bioplastics [7-9]. Given these successes and the immense potential of the field, though, why have many industrial players been slow to adopt and commit to synthetic biology? One reason pertains to the relative infancy of the field. Additional and substantial investment of time and money are still required for synthetic biology to reach the stature of a 'mature discipline.' After all, when it comes to producing renewable biochemicals, the complexity and elegance of biocatalyst design often matters less than the key metrics of titre, yield and productivity. Economics will always dictate the bottom line in any bioprocess.

Nevertheless, synthetic biology will continue to make important inroads into the 'green chemicals' industry. Perhaps the best example is the microbial production of artemisinin, a potent antimalarial drug, by Jay Keasling and co-workers at the University of California, Berkeley, USA. Artemisinin is a natural plant product derived from the leaves and flowers of Artemisia annua. The traditional cultivation and harvesting methods have long prevented economical large-scale production, thereby greatly limiting the availability of a life-saving drug to poor and rural communities in Africa and southeast Asia. The Keasling team developed a yeast-based

...achieving functionality of a
newly engineered or discovered
pathway is merely the first step
in the production of novel green
chemicals

microbial 'biofactory' for artemisinic acid by re-engineering the yeast mevalonate isoprenoid biosynthesis pathway to include a synthetic gene, amorpha-4,11-diene synthase. This new part enhances and balances metabolic flux through the pathway, thereby improving the overall function of the biosynthetic device. Systematic expansion and optimization of the pathway by the incorporation of additional plantderived enzyme parts, such as a cytochrome P450 monooxygenase from Artemisia annua, increased the titre of artemisinic acid. The laboratory work is now being translated to an industrial scale. Together with Sanofi, Amyris Biotechnologies, a start-up company founded in 2003 by the Keasling laboratory, is scaling up the bioproduction process to 50-60 tonnes per year by 2014 , which is enough for 80-150 million treatments.

Synthetic biology is also beginning to make an impact on the commodity chemicals market. This year, for example, Genomatica, working with DuPont Tate \& Lyle, produced 2,000 tons of 1,4-butanediol, a non-natural bioplastic monomer, directly from sugar in a single 5-week run. Genomatica has used pathway-identification algorithms and genome-scale metabolic models to optimize their microbial biocatalysts [10].

Following in the wake of Amyris and Genomatica, entrepreneurial scientists are spearheading synthetic biology's foray into industrial biotechnology. This new crop of synthetic biology ventures includes OPX Biotechnologies, Lygos, Ginkgo Bioworks, Manus Biosynthesis and Pivot Bio. These and other start-up companies continue to establish synthetic biology's role in 'green chemistry'.

$\mathrm{N}$ otwithstanding such progress, much more remains to be done to improve our understanding of intrinsic microbiological properties and optimum strategies for the implementation of synthetic pathways. For example, mutation and evolution remain key aspects: since human-designed functions tend to impose a metabolic burden on the host, natural selection can result in population dominance of non-productive mutants and decrease overall productivity. This could become a serious problem, especially for continuous cultures or batch fermentations over long periods of time. Mutability is helpful for the preliminary design as it expands choices of biological components

\author{
Sidebar A | Further reading
}

Metabolic engineering to increase productivity: Alper H, Moxley J, Nevoigt E, Fink GR, Stephanopoulos G (2006) Engineering yeast transcription machinery for improved ethanol tolerance and production. Science 314: 1565-1568 $\mathrm{Na}$ D, Yoo SM, Chung H, Park H, Park JH, Lee SY (2013) Metabolic engineering of Escherichia coli using synthetic small regulatory RNAs. Nat Biotechnol 31: 170-174

Wang HH, Isaacs FJ, Carr PA, Sun ZZ, Xu G, Forest CR, Church GM (2009) Programming cells by multiplex genome engineering and accelerated evolution. Nature 460: 894-898

Enzyme discovery and protein design: Romero PA, Arnold FH (2009) Exploring protein fitness landscapes by directed evolution. Nat Rev Mol Cell Biol 10: 866-876

Stemmer WP (1994) Rapid evolution of a protein in vitro by DNA shuffling. Nature 370: 389-391

Röthlisberger D, et al (2008) Kemp elimination catalysts by computational enzyme design. Nature 453: 190-195

\section{Pathway refactoring:}

Temme K, Zhao D, Voigt CA (2012) Refactoring the nitrogen fixation gene cluster from Klebsiella oxytoca. Proc Natl Acad Sci USA 109: 7085-7090

Metabolic channelling:

Conrado RJ et al (2012) DNA-guided assembly of biosynthetic pathways promotes improved catalytic efficiency. Nucleic Acids Res 40: 1879-1889

Delebecque CJ, Lindner AB, Silver PA, Aldaye FA (2011) Organization of intracellular reactions with rationally designed RNA assemblies. Science 333: 470-474

Dueber JE, Wu GC, Malmirchegini GR, Moon TS, Petzold CJ, Ullal AV, Prather KL, Keasling JD (2009) Synthetic protein scaffolds provide modular control over metabolic flux. Nat Biotechnol 27: 753-759

and systems, but it must be minimized in final designs to maintain strain robustness. For example, synthetic genetic circuits can be designed to minimize loss-of-function mutations or eliminate non-productive mutants. Such a 'synthetic selection' concept can also be applied to develop safe engineered strains, which cannot survive beyond a controlled environment.

Modular and standardized elements are particularly useful for 'green chemistry' applications to rapidly and reliably exchange pathway elements, such as enzymes, promoters and even entire metabolic pathways, in a 'plug-and-play' manner. Such flexibility supports the rapid 
prototyping of new strains and, ultimately, the systematic optimization of microbes as adaptable 'biorefineries' with scalable productivity. However, truly interchangeable parts and standard production platforms do not yet exist. Despite the establishment of parts libraries and increased understanding of context effects on gene expression and production hosts, universal parts, devices and systems remain a future ambition. The engineering of minimal genome organisms, along with advancements in refactoring approaches, will aid in the engineering of standard production hosts, in which context effects could be better predicted and crosstalk between introduced pathways and chassis should be less severe.

Lastly, as the field continues to mature, it is critical that the development of synthetic biology in research and industrial applications is accompanied with active public engagement. Discussions surrounding biosecurity, biosafety, ethics, intellectual property and environmental issues must continue and are being facilitated through educational activities, such as the International Genetically Engineered Machine competition, as well as within the political realm. The experience of genetically modified crops in Europe has demonstrated the importance of garnering public support for nascent areas of research to avoid a public backlash that could jeopardize synthetic biology's potential in green chemistry.

\section{ACKNOWLEDGEMENTS}

D.R.N. is supported by the National Science Foundation (CBET1159200, CBET 1067684), the Department of Energy, ASU LightWorks, and seed funding from Arizona State University. T.S.M. is supported by the Bill \& Melinda Gates Foundation, the National Science Foundation (MCB1331194), and the International Centre for Advanced Renewable Energy \& Sustainability (I-CARES).

\section{CONFLICT OF INTEREST}

The authors declare that they have no conflict of interest.

\section{REFERENCES}

1. Nikolau BJ, Perera MADN, Brachova L, Shanks B (2008) Platform biochemicals for a biorenewable chemical industry. Plant / 54: 536-545

2. Prather KL, Martin CH (2008) De novo biosynthetic pathways: rational design of microbial chemical factories. Curr Opin Biotechnol 19: 468-474

3. Farmer WR, Liao JC (2000) Improving lycopene production in Escherichia coli by engineering metabolic control. Nat Biotechnol 18: 533-537

4. Zhang F, Carothers JM, Keasling JD (2012) Design of a dynamic sensor-regulator system for production of chemicals and fuels derived from fatty acids. Nat Biotechnol 30: 354-359

5. Bayer TS, Widmaier DM, Temme K, Mirsky EA, Santi DV, Voigt CA (2009) Synthesis of methyl halides from biomass using engineered microbes. I Am Chem Soc 131: 6508-6515

6. Nam H, Lewis NE, Lerman JA, Lee DH, Chang RL, Kim D, Palsson BO (2012) Network context and selection in the evolution to enzyme specificity. Science 337: 1101-1104

7. Immethun $\mathrm{CM}$, Hoynes-O'Connor AG, Balassy A, Moon TS (2013) Microbial production of isoprenoids enabled by synthetic biology. Front Microbiol 4: 75

8. Adkins J, Pugh S, McKenna R, Nielsen DR (2012) Engineering microbial chemical factories to produce renewable "biomonomers". Front Microbio/ 3: 313

9. Lee JW, Kim HU, Choi S, Yi J, Lee SY (2011) Microbial production of building block chemicals and polymers. Curr Opin Biotechnol 22: 758-767

10. Yim H et al (2011) Metabolic engineering of Escherichia coli for direct production of 1,4-butanediol. Nat Chem Biol 7: 445-452
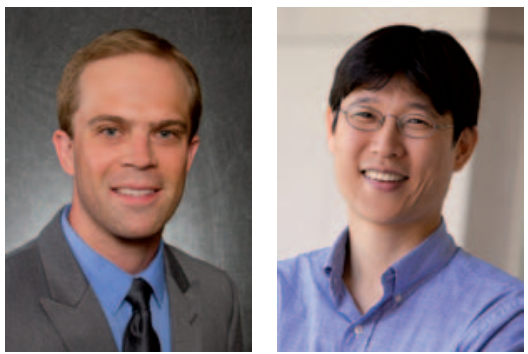

David Nielsen is at the School for Engineering of Matter, Transport, and Energy, Arizona State University in Tempe, Arizona, USA.

E-mail:david.r.nielsen@asu.edu Tae Seok Moon is at the Department of Energy, Environmental \& Chemical Engineering, Washington University in St Louis, Missouri, USA.

E-mail:tsmoon@seas.wustl.edu

EMBO reports (2013) 14, 1034-1038; published online 8 November 2013; doi:10.1038/embor.2013.178 